\title{
Influence of irradiation on the dynamics of changes in the mechanical properties of the nuclear reactor vessel
}

\author{
Yulia S. Bakhracheva* \\ Department of Information Security, Institute of priority technologies, Volgograd State University, \\ 400062 Volgograd Prosp. Universitetsky 100, Russian Federation
}

\begin{abstract}
Nuclear power plants are important generating units of the energy system worldwide. In the normal mode, nuclear power plants are absolutely safe, but emergency systems with radiation emissions have a devastating impact on the environment and public health. Despite the introduction of technologies and automatic monitoring systems, the threat of a potentially dangerous situation remains. The reactor vessel is the main object of activities to ensure the safety of nuclear power plants. One of the problems of ensuring the safety of nuclear power plant reactor vessels is the prediction of the level of crack resistance of reactor steels. The paper shows the possibility of estimating the neutron irradiation level on the nature of the temperature dependence of $\mathrm{K}_{\mathrm{IC}}$. The prediction of the influence of radiation damage on the fracture toughness of the reactor steel can be obtained on the basis of the results of tests of small cylindrical samples with annular notches.
\end{abstract}

\section{Introduction}

In the normal mode, nuclear power plants are absolutely safe, but emergency situations with radiation emissions have a devastating impact on the environment and public health. Despite the introduction of technologies and automatic monitoring systems, the threat of a potentially dangerous situation remains.

The Russian nuclear power development program provides for the continuation of the operation of nuclear power plants after the end of the design thirty-year service life by performing a set of works that ensure the safety of their further operation.

The problem of ensuring the design life of nuclear power plant units and their extension is currently very relevant not only in Russia but also in many countries around the world. The solution to this problem allows to increase the efficiency of nuclear power plants without additional investments to replace the decommissioned capacity.

The main structural element of the power unit with a nuclear power plant, which determines its life, is the reactor vessel [8]. If other structural elements (steam generator, pipelines, heaters, etc.) can be replaced or repaired in case of detection of damage in them, the reactor vessel shall perform its functions without failure during the entire project or

*Corresponding author: bakhracheva@volsu.ru 
post-project (in case of extension) service life. It is very important to be able to adequately assess the real life of reactor vessels in comparison with the design and manage it, i.e. to increase it if necessary by applying a number of special measures.

The reactor vessel resource is determined by the characteristics of the material, manufacturing methods and operating conditions. The main factors affecting the reactor vessel during operation and determining its life are: internal pressure, forces on the pipes from the attached pipelines, temperature impact, radiation.

The first three factors often are interrelated, and you can talk about the temperature and force loading of the casing. These loads can be both static and dynamic and cause stresses and strains in the structure and damage accumulation in stress concentration zones. The degree of damage at the end of life depends on the number of repetitions of different types of modes during the design life.

In parallel to the process of accumulation of damage from thermal power loads, the properties of the material of reactor vessels are degraded from temperature and radiation exposure, which is expressed in its hardening and embrittlement. It is known that these changes depend on the energy of irradiating particles, their integral dose and temperature of irradiation.

The reactor vessel is the main object of activities to ensure the safety of nuclear power plants. One of the problems of ensuring the safety of nuclear power plant reactor vessels and predicting their residual life is the prediction of the level of crack resistance of reactor steels. And it is important to know how the crack resistance varies depending on the temperature and how this dependence changes when irradiating the material. Therefore, from the point of view of the safety problem of nuclear power plants, the main task of mechanics and physics of materials of reactor vessels should be considered the prediction of the impact of radiation damage on the fracture toughness of these materials.

\section{Mathematical model}

The existing standard approaches to this problem (the use of the "Master curve" approach, the determination of the critical temperature displacement for a given level of Charpy impact viscosity) are essentially empirical, so they are not applicable for conditions when, along with the shift of the temperature dependence of the fracture toughness or impact toughness, there is a change in the form of temperature dependence of these characteristics, as well as the transition to new grades of reactor steels.

The most promising approach to predict crack resistance of irradiated reactor materials, which is currently intensively developed, is based on a local approach to the analysis of viscous and brittle fracture. The main idea of the local approach is to describe the process of growth of the main crack based on the analysis of the metal fracture in the vicinity of its top. As shown in $[1,2]$, the fracture at the top of the main crack is initiated in extremely small volumes, the value of which, as a rule, does not exceed $1 . . .50 \mathrm{~mm}^{3}$.

In this regard, in the works [1-3] was proposed a fundamentally different approach to the solution of the problem under consideration, which is to calculate the probability of failure based on the analysis of elementary acts of destruction associated with the formation and loss of stability of embryonic cracks. In the framework of this approach it is possible to Express the parameters of the fracture probability function through constants that are invariant to the form of the stress-strain state and characterize the properties of the metal. This creates the conditions for using the proposed version of the local approach as a tool to study the effect of changes in the crack resistance of steel after radiation exposure.

According to the modern concepts of fracture physics, the growth of a main crack in the metal occurs by the destruction of the metal in the vicinity of the top of a macro crack as a result of the formation and loss of stability of embryonic cracks. These embryonic cracks 
occur in the local area before the top of the main crack. The conditions for the formation and buckling of embryo cracks are controlled by the local stress and strain field created by the main crack. In this case, the key problem in the description of fracture is to determine the probability of loss of stability of one nucleated crack $p_{0}$ at a given stress level $\sigma_{F}$. The fracture probability function can be obtained from the analysis of the formation and buckling of nucleated cracks in the metal.

In the vicinity of the main crack or notch, the stress and strain fields are distributed nonuniformly. Currently, the finite element method is successfully used for their calculation. The expression for the probability of $p_{i}$ loss of stability of at least one crack in the volume of metal bounded by one finite element is determined by the formula:

$$
p_{i}\left(\sigma_{F}\right)=1-\left[1-p_{0}\left(\sigma_{F}\right)\right]^{V_{i} \rho_{i}}
$$

where $V_{i}$ the volume of the finite element; $\rho_{i}$ is the density of nuclei cracks formed in this volume of metal.

Condition of brittle fracture of the entire sample with a main crack or incision consisting of $M$ finite elements:

$$
P_{f}=1-\prod_{i=1}^{M}\left[1-p_{i}\left(\sigma_{F}\right)\right]
$$

where $P_{f}$ is a tolerance on the probability of the event in question.

In the analysis of temperature dependences of crack resistance, the average value of tolerances for the probability of failure $P_{f}=0.5$ is of interest.

It should be noted that the length of the embryo cracks is not arbitrary, its size is given by the size of the metal microstructure. Depending on the chemical composition of the steel and its heat treatment it can be the size of martensite or bainite package, ferritic grain, carbide diameter. This property of the embryo cracks is very important, because it allows us to understand how the size of the microstructural components determine the level of brittle strength of steel $R_{M C}$.

A very important property of germ cracks, which is not taken into account in existing models, is that the density of these cracks is not a constant. It depends on the plastic deformation prior to failure and the test temperature. In a simplified form, the effect of plastic deformation on the density of the embryo cracks can be described by the formula:

$$
\rho=k_{\rho} \int_{\varepsilon_{c}}^{\varepsilon_{R}} f(\varepsilon) d \varepsilon
$$

where $\rho$ is the density of nucleated cracks; $f(\varepsilon)$ is a function of the density distribution of microplastic deformations in a polycrystal; $k_{\rho}$ is a coefficient that depends on the probability of formation of a blocked dislocation cluster of critical length; $\varepsilon_{c}$ is the critical value of the intensity of microplastic deformation necessary for the formation of embryonic cracks; $\varepsilon_{R}$ is the critical value of the microplastic deformation, at which, instead of the formation of an embryonic crack, stress relaxation occurs at the top of the dislocation cluster.

As noted in $[2,3]$, the dependence of the density of the embryo cracks on the magnitude of plastic deformation and the test temperature is a distinctive feature of brittle (quasibrittle) destruction of metals in comparison with the destruction of brittle materials such as glass. This should lead to the influence of plastic deformation on the magnitude of the scale effect, which is a characteristic feature of local destruction. Before the incision or acute crack, the value of plastic deformation varies in a wide range, so the influence of plastic 
deformation on the magnitude of the scale effect and is of fundamental importance in the destruction of the metal under stress concentration conditions.

The paper proposes a statistical model that describes the brittle fracture of polycrystalline metals based on the above properties of nucleated cracks. Within this approach, the key expression for the local approach is described by the formula:

$$
p_{0}\left(\sigma_{F}\right)=0,5 \int_{\sigma_{c}^{\min }}^{\sigma_{c}^{\max }} f_{1}\left(\sigma_{c}\right)\left[1-\left(\frac{\sigma_{c}-\sigma_{F}}{\sqrt{2} I_{\sigma} \sigma_{F}}\right)\right] d \sigma_{c}
$$

where $I_{\sigma}$ is the coefficient of variation of the maximum tensile microstresses; $\sigma_{c}^{\min }$ and $\sigma_{c}^{\max }$ - the minimum and maximum values of the critical microstrain of the loss of stability of the embryo crack of random orientation and random length; $f_{1}\left(\sigma_{c}\right)$ is a function of the density distribution of these stresses.

\section{Discussion}

In this paper, on the example of cabinet reactor steel $15 \mathrm{Cr} 2 \mathrm{MoVA}$ shown the ability to predict the effects of neutron irradiation on the level and nature of the temperature dependence of $K_{I C}$. Data on the mechanical properties of this steel in the initial state and after neutron irradiation are taken from [4].

Using experimental data for the initial fracture toughness of $K_{I C}$ steel at temperatures from 77 to $273 \mathrm{~K}$ and the brittle strength of steel $R_{M C}=1500 \mathrm{MPa}$, constants were determined depending on (3). The values of other constants are weakly dependent on the microstructure of steel, so the data for iron were used.

On the basis of these data, the values of the fracture toughness of the reactor steel in the initial state are calculated, and the temperature dependences of the fracture toughness with a predetermined fracture probability of 0.5 for fluence $10^{19}$ are predicted.

According to the data obtained, the results of computer simulation are in good agreement with the experimental dependence of crack resistance at fluence of $10^{19}$ neutrons per $\mathrm{cm}^{2}$.

\section{Conclusion}

The proposed version of the local approach is based on the modeling of macro-destruction based on the analysis of elementary acts of the process of brittle metal fracture. This allows it to be used for solving both fracture mechanics and materials science problems. To solve the material science problems, it is possible to simulate the effect of the steel microstructure parameters on the level of its crack resistance in the initial and irradiated states. The application of the local approach for solving problems of fracture mechanics that are related to predicting changes in fracture toughness of reactor pressure vessel steels after neutron irradiation, involves the use of information about the standard properties of the irradiated steel to be determined by smooth standard specimens. The physical meaning of the procedure for selecting the type of samples and the method of their testing is that in addition to the standard properties (yield strength and strain hardening index) of irradiated steel, it is necessary to obtain information about the level of its brittle strength $R_{M C}$, as well as the sensitivity of the brittle fracture stress to the volume change (scale effect). In the most general case (new steel grade, welded seams, large fluences, etc.) this information can be obtained on the basis of the test results of small cylindrical specimens with annular cuts. 


\section{References}

1. Ju. Bakhracheva, IJCCSE, 9 (3), 21 (2013)

2. A. Baron, J. Bakhracheva, Mechanics, 66 (4), 31 (2007)

3. Yu. Bakhracheva, Science Journal of Volgograd State University. Technology and Innovations, 11, 27 (2014) doi: 10.15688/jvolsu10.2014.2.4

4. Y. Bakhracheva, A. Vasilyev, T. Petikova, J. Materials science forum, 870, 545 (2016) doi: 10.4028/www.scientific.net/MSF.870.545

5. A. Baron, J. Bakhracheva, A. Osipenko, Mechanics, 67, 33 (2007)

6. V. I. Shapochkin et al. Metal Science and Heat Treatment, 52, 413 (2011)

7. L. M. Semenova et al. Metal Science and Heat Treatment, 55, 34 (2013)

8. V. M. Khokhlov, Russia Engineering Research, 16, 11 (1996)

9. A. V. Vasilyev, et al., Vestnik Volgogradskogo gosudarstvennogo universiteta. Seriya 10, Innovatsionnaya deyatelnost [Science Journal of Volgograd State University. Innovation Activity], 10, 26 (2014)

10. A. V. Vasilyev et al., Procedia engineering, 150, 312 (2016) doi:10.1016/j.proeng.2016.07.017

11. A. Vasilyev, Y. Bakhracheva, IOP Conf Series: Journal of Phisics: Conf. Series, 1050, 012094 (2018) doi: 10.1088/1742-6596/1050/1/012094

12. Y. Bakhracheva, IOP Conf Series: Journal of Phisics: Conf. Series, 1050, 012006 doi.org/10.1088/1742-6596/1050/1/012006 (2018) 